\title{
Point of care diaphragm ultrasound in mechanically ventilated children: A predictive tool to detect extubation failure
}

\author{
Gazi Arslan ${ }^{1}$, tolga besci ${ }^{1}$, and Murat Duman ${ }^{2}$ \\ ${ }^{1}$ Dokuz Eylül University \\ ${ }^{2}$ Dokuz Eylul University Faculty of Medicine
}

February 12, 2022

\begin{abstract}
Background: Children should be weaned from the ventilator once their clinical condition improves. Extubation failure is associated with poorer clinical outcomes in children. Predictive indicators of successful extubation are needed. This study aims to evaluate the predictive value of ultrasonographic diaphragm imaging could help predict weaning success. Methods: In this prospective, observational study conducted between March and December 2021, children between 1 month and 10 years of age who were mechanically ventilated for more than 48 hours were included. Diaphragm ultrasound (DUS) examinations were performed at the end of 2-hour extubation readiness test (ERT). The end-inspiratory thickness, end-expiratory thickness, diaphragmatic thickening fraction, diaphragmatic excursion, inspiratory slope and expiratory slope were evaluated. Results: Twenty-four (60\%) patients were successfully extubated, while 16 (40\%) required invasive or non-invasive mechanical ventilation support which were classified as failed extubation group. Three of the sixteen patients in the failed extubation group required re-intubation. Diaphragm thickening fraction was significantly greater in the successful weaning group $(55,05 \pm 23,75 \%$ vs. $30,9 \pm 10,38 \%)(\mathrm{p}<0,001)$. Diaphragm excursion was significantly greater in the SW group $(14 \pm 4,4 \mathrm{~mm}$ vs $11,05 \pm 3,25 \mathrm{~mm})$ $(\mathrm{p}<0,001)$. DTF and DE were found to have a sensitivity and specificity of $91.67 \%, 87.50 \%$, and $83.33 \%, 81.25 \%$, respectively. Conclusion: Diaphragm ultrasound is a feasible and promising tool to guide physicians during weaning from IMV. Among all DUS measurements, the DE and DTF indexes showed better performance in extubation failure than other diaphragmatic parameters.
\end{abstract}

\section{Introduction}

Invasive mechanical ventilation (IMV) with positive pressure is widespread in pediatric intensive care units (PICUs), with at least one-third of children being mechanically ventilated. ${ }^{1,2}$. Prolonged IMV is associated with ventilator-related injury, increased nosocomial infection risk, longer sedation requirement ${ }^{3}$ and diaphragmatic atrophy ${ }^{4}$. Ultimately, children should be weaned from the ventilator once the clinical condition improves. However, the optimal timing of extubation is not easy to determine, considering both early and late extubation may affect clinical outcomes. Extubation failure is associated with poorer clinical outcomes in children, with a reported rate of 2.7 percent to $22 \% .^{3,5,6}$. Children's weaning practices vary depending on hospitals, staff, and resources. Therefore, predictive indicators of successful extubation are needed. Several indices have been developed to predict weaning success and extubation failure, including the Rapid Shallow Breathing Index $(\mathrm{RSBI}=\mathrm{f} / \mathrm{Vt})$; Maximal Inspiratory Pressure (MIP), Compliance Resistance Oxygenation Pressure Index (CROP); Volumetric Capnography; and Extubation Readiness Test ${ }^{7}$ (ERT). However, none of the above indices have been widely used in PICU practice except ERT ${ }^{8-10}$.

Due to the suppression of inspiratory effort, diaphragmatic atrophy occurs quickly in mechanically ventilated children, resulting in ventilator induced diaphragmatic dysfunction (VIDD), which is related to difficulties weaning from IMV, prolonged duration of stay, and other complications ${ }^{4,11,12}$. Additionally, high ventilator mandatory breathing rates, corticosteroids, and neuromuscular blockers aggravate VIDD ${ }^{13}$. 
With the extensive use of ultrasound in critical care settings, detecting VIDD and predicting weaning outcomes using ultrasonographic diaphragm imaging have been clinical research hotspots in recent years. Several adult studies demonstrated that some structural indexes such as excursion, thickness and thickening fraction of diaphragm obtained with the point of care diaphragm ultrasound (DUS) have good predictive values on successful extubation ${ }^{14,15}$. Although various research has demonstrated that DUS may be used to examine children's diaphragm functions ${ }^{16}$ and predict extubation failure ${ }^{17,18}$, there is a dearth of evidence regarding its use in weaning procedures.

This study aims to evaluate the predictive value of DUS in weaning outcomes in mechanically ventilated children and develop a more reliable tool for its use in pediatric intensive care units.

\section{Materials and Methods}

\section{Population and Study Design}

This prospective, observational study was conducted in our medical/surgical PICU between March and December 2021, with the approval of our institutional review board (2022/03-10). Written informed consent was obtained from the parents at the time we enrolled the patient. Children between 1 month and 10 years of age who were mechanically ventilated for more than 48 hours via an endotracheal tube in the PICU were screened for inclusion. The patients were assessed daily for ventilator weaning and extubation according to our institutional weaning protocol. Patients who met the following criteria were recruited: 1) recovery from primary disease-causing mechanical ventilation, 2) adequate spontaneous breathing and effective coughing, 3) increasing consciousness, 4) hemodynamic stability with minimal vasoactive support, $5)$ oxygenation index $<6,6$ ) lack of excessive tracheal secretion (less need for endotracheal suctioning), 7) adequate gas exchange with positive end-expiratory pressure (PEEP) $<8 \mathrm{~cm} \mathrm{H} 2 \mathrm{O}$ and fractional oxygen concentration ( $\mathrm{fiO} 2)<60 \%$. The exclusion criteria were as follows: 1) detected diaphragm paralysis, 2) underlying neuromuscular disease and chronic respiratory failure, 3) a current tracheostomy, 4) pleural effusion, pneumothorax or pneumomediastinum.

\section{Weaning procedure}

The patients were assessed daily for weaning. If they fit weaning criteria, ERT with pressure support ventilation (PSV) for 2 hours was performed according to our institutional protocol. The PSV settings were set as: PEEP at $5 \mathrm{~cm} \mathrm{H} 2 \mathrm{O}$, PS adjusted minimally to overcome endotracheal tube resistance and generate a tidal volume of $4-8 \mathrm{ml} / \mathrm{kg}$, and $\mathrm{fO} 2$ at $40 \%$. The ERT failure criteria were as follows: 1) clinical findings: diaphoresis, nasal flaring, increased work of breathing, tachypnea ( $>50 \%$ increase compared to baseline), tachycardia ( $>20 \%$ increase compared to baseline), apnea, arrythmia, hypotension, increase of $>10 \mathrm{mmHg}$ of end-tidal carbon dioxide compared to the baseline value 2) laboratory findings:, arterial blood $\mathrm{pH}<7.32$, decrease in arterial $\mathrm{pH}$ by 0.07 or more compared to baseline, decrease in $\mathrm{SpO} 2$ more than $5 \%^{3}$. Patients who did not fail ERT had a diaphragmatic ultrasound and were extubated right away. Successful weaning (SW) was defined as patients who did not require mechanical ventilation (invasive or non-invasive) within 48 hours of extubation, whereas failed extubation (FE) was defined as patients who required re-intubation or non-invasive mechanical ventilation within 48 hours of extubation. Another intensivist who was blinded to DUS measurements made the decision regarding the need for reintubation or non-invasive ventilation following extubation.

\section{Diaphragmatic ultrasound measurement}

All the ultrasonographic measurements were made by the same pediatric intensivist (who was specially trained) using a portable Esaote MyLab Omegaß system equipped with a 4-15 Mhz linear transducer for diaphragmatic thickness (DT), diaphragmatic thickening fraction (DTF), and a 6-10 Mhz convex transducer for diaphragmatic excursion (DE), inspiratory slope (IS), and expiratory slope (ES). We evaluated right diaphragm since previous studies demonstrated that there was no difference in sonographic measurements between the two sides and right diaphragm was easier to assess ${ }^{19,20}$. All patients were supine with the head of the bed elevated $30^{\circ}$. Measurements were obtained at the end of the two-hour ERT. First, as in previous 
studies $^{21}$, the linear transducer in B-mode was placed between the anterior axillary and midaxillary lines, in the $9^{\text {th }}$ and $10^{\text {th }}$ intercostal spaces. The diaphragm is depicted as a three-layer structure in this image, with two parallel echoic lines (pleural and peritoneal lines) and a hypoechoic structure between them (the muscle itself). DT was measured three times, both at the end of inspiration (DTi) and expiration (DTe). The percent change in DT between end-expiration and end-inspiration (DTF) was calculated as (DTi-DTe/Dte) x 100 (Figure 1-A, B).

Second, using the right hepatic lobe as an acoustic window, the 2-5 Mhz transducer was placed immediately below the right costal margin along the mid-clavicular line and directed medially, cephalad, and dorsally. The posterior third of the diaphragmatic muscle was visualized using this technique. Then, M-mode is used to visualize the diaphragm's motion during the respiratory cycle. The diaphragm moves caudally toward the probe during inspiration; it moves cranially away from the probe during expiration. Three respiratory cycles were recorded, and the average was calculated. DE is measured on the vertical axis of the trace from the baseline to the point of maximum height of inspiration ${ }^{22}$ (Figure 1-C, D). Three serial respiration cycles were recorded for each patient, and mean values for each diaphragmatic parameter was calculated. Intraobserver variability of ultrasonic measurements were less than $10 \%$.

\section{Data collection}

Demographic and clinical data were collected from each patient's electronic records, including age, sex, underlying disease, indication for IMV, Pediatric Index of Mortality III (PIM-3), Pediatric Risk of Mortality IV (PRISM-4), duration of IMV, maximum oxygenation saturation index, ventilatory parameters during ERT (respiratory rate, tidal volume, dynamic compliance, MIP), need for inotrope infusion, maximum VIS score, treatments used (aminoglycoside, corticosteroid, neuromuscular blockage), length of PICU stay, and mortality. The DTi, DTe, DTF, DE, IS, and ES values were also recorded.

\section{Statistical analysis}

The data analysis was performed using SPSS 25.0 software (IBM, Armonk, NY). The count data was expressed in the form of numbers (percentage). The independent sample t-test was used to compare continuous, normally distributed data expressed as mean \pm standard deviation; the Mann Whitney $\mathrm{U}$ test was used to compare non-normally distributed data expressed as median (interquartile range). Pearson's Chi-squared or Fisher's exact tests were used to compare categorical data. Kruskal Wallis test followed by Tamhane's posthoc pairwise comparisons were used to compare DTF and DE values of groups according to post-extubation intervention requirements. All tests were two-tailed, and statistical significance was defined as a p-value less than 0.05. The receiver operating characteristic analysis (ROC) was used to determine the predictive value of each index in this study. The optimal threshold, along with its sensitivity and specificity, was determined using the Yuden index.

\section{Results}

Between March and December 2021, 40 patients were enrolled, and DUS examinations were performed (figure 2). Within 48 hours, $24(60 \%)$ of patients were successfully extubated, while $16(40 \%)$ required invasive or non-invasive MV support. Three of the sixteen patients in the FE group required re-intubation (7.5\%), and thirteen received non-invasive MV (32.5\%). Between the SW and FE groups, there were no statistically significant differences in age, sex, PIM-3, PRISM-4, need for inotrope infusion, indication for IMV, maximum OSI, and treatments used. The FE group had a longer duration of inotrope infusion days, a longer duration of IMV, and a longer length of stay in the PICU than the SW group (table 1).

Diaphragm measurements of the SW and FE groups are also shown in table 1 . While DTi and DTe did not differ significantly between the two groups, DTF was significantly greater in the SW group $(55,05 \pm 23,75 \%$ vs. $30,9 \pm 10,38 \%)(\mathrm{p}<0,001)$. DE was significantly greater in the $\mathrm{SW}$ group $(14 \pm 4,4 \mathrm{~mm}$ vs $11,05 \pm 3,25$ $\mathrm{mm})(\mathrm{p}<0,001)$, but there was no significant difference in IS or ES between the two groups. When DTF and DE were compared in terms of post-extubation intervention requirements, while DTF was significantly different between those who required reintubation and those who required NIV ( $\mathrm{p}=0.021)$, there was no 
significant difference in terms of $\mathrm{DE}(\mathrm{p}=0.620)$ (table 2).

The ROC curves for DTF and DE are shown in figure 3. After performing a ROC analysis and calculating the AUC, the AUCs (95\% CI) for DTF and DE were 0.962 (0.911-1) and 0.880 (0.762-0.998), respectively. DTF and DE had optimal cutoff points of 40.5 and $12.15 \mathrm{~mm}$, respectively. Of the 26 patients who were successfully extubated, 22 had a DTF greater than $40.5 \%$. Of the 16 who failed extubation, 14 had a DTF less than $40.5 \%$. DTF and DE were found to have a sensitivity and specificity of $91.67 \%, 87.50 \%$, and $83.33 \%, 81.25 \%$, respectively. The positive and negative predictive value of DTF and DE were also shown in table 3.

\section{Discussion}

Point of care ultrasound is increasingly used in pediatric critical care settings. Recently, ultrasound has been used to assess the diaphragm functions ${ }^{17}$, identify $\operatorname{VIDD}^{20}$, pneumonia and bronchiolitis ${ }^{23}$ and evaluate diaphragm motions during spontaneous breathing trials ${ }^{21}$. When DTF and DE were determined using DUS in the zone of apposition at the end of the ERT, we found a significant difference between the SW and FE groups. Although, these findings are similar to previous studies evaluating the role of DUS on the weaning policy, this is the first pediatric study evaluating DUS at the end of the ERT. Also, this study showed that DUS findings were significantly differ between NIV requirement and reintubated patients who were considered as extubation failure. Additionally, the FE group had a statistically significantly longer IMV duration, indicating that prolonged IMV duration was associated with increased diaphragmatic atrophy.

Extubation failure is reported as high as $33 \%$ in some studies ${ }^{6}$. The failure rate in our study was $40 \%$, which was significantly higher than the rate in other studies. This difference could be explained by the fact that among all the patients included only three (7.5\%) required reintubation and the tendency in our PICU practice to use NIV following extubation. With such high rate of extubation failure and NIV requirement, it is obvious that, more reliable tools are needed to anticipate weaning outcomes and identify indications for NIV treatment. RSBI has been demonstrated to be accurate in predicting extubation failure in adults $^{24}$. However, this index reflects the contribution of all inspiratory muscles, rather than the diaphragm. In addition, due to different respiratory rates in different ages and the tidal volume is weight-related, there is no constant threshold for RSBI in children. CROP Index found by Thiagarajan et al ${ }^{9}$, was a good predictor for successful extubation, pediatric studies demonstrated that this index did not reliably predict extubation failure in children ${ }^{25}$. Although there are studies showing that volumetric capnography can also predict the successful extubation in children, it is not widely used in clinical practice ${ }^{10}$.

In mechanically ventilated children, inspiratory muscles especially diaphragm weakens and VIDD occurs rapidly. Within the first week of IMV, the diaphragm's thickness decreases by more than $10 \%$, and an increasing percentage of muscle fat degrades muscle quality ${ }^{17}$. After a few hours of ERT, the breathing pattern, which is usually normal at the start, deteriorates. As such, we sought to determine whether changes in diaphragm function at the conclusion of the ERT are an accurate predictor of successful extubation.

Yoo et al, compared the predictive values of DE and DTF in adults, found that best prediction thresholds for DE and DTF were 10-15 mm and 20-36\% respectively; the sensitivity and specificity for DE and DTF were $0.86-0.79,0.90-0.80$, respectively ${ }^{26}$. In our study, DE of children was similar to adults: the optimal cut-off was $12.15 \mathrm{~mm}$, DTF of children was higher than adults: the optimal cut-off was $40.5 \%$. This is consistent with the clinical severity difference between FE groups between two studies. They had more severe disease, and six required tracheotomies, while five died during their intensive care unit stay. However, only three of our patients required reintubation, and the remaining thirteen were followed with NIV without reintubation. Lee et al, found that DTF was significantly different between the successful and failed extubation groups in children and a DTF value of $<17 \%$ was associated with extubation failure ${ }^{17}$. Also, Ijland et al, found that over $90 \%$ of children were successful extubated with a median DTF of $15.2 \%^{27}$. In both studies above, there were only 3 patients in the failed extubation groups, and those 3 patients were also required reintubation. However, children who required reintubation had a DTF value of less than $20 \%$ in our study.

Our study had some limitations. First, our population was relatively small, despite the fact that we included 
a comparable number of patients as previous studies ${ }^{17,18}$. Specifically, only three patients in the FE group were reintubated, which precluded us from revealing the DUS findings regarding the difference between NIV requirement and reintubation. It has been demonstrated that the use of NIV reduces the need for reintubation in children ${ }^{28}$. It is necessary to conduct larger, multicenter studies comparing the DUS findings in terms of NIV requirement, reintubation, and successful extubation. Second, because no reference for DTF and DE in children has been established, and in the absence of initial DUS findings prior to IMV, it is difficult to determine whether the included children's initial diaphragmatic function was abnormal or not. Third, the heterogeneity of the IMV indication limits the generalization of the study results on general PICU patients.

\section{Conclusion}

Diaphragm ultrasound is a feasible and promising tool to guide physicians during weaning from IMV. Among all DUS measurements, the DE and DTF indexes showed better performance in extubation failure than other diaphragmatic parameters.

\section{Acknowledgement}

This research did not receive any specific grant from funding agencies in the public, commercial, or not for profit sectors.

\section{Conflict of Interest}

All the authors declare that there are no conflict of interest.

\section{Author Contribution}

Gazi Arslan: conceptualization (equal); data curation (equal); formal analysis (equal); writing original draft (equal); writing review and editing (equal). Tolga Besci: conceptualization (equal); data curation (equal); methodology (equal); formal analysis (equal); writing review and editing (equal). Murat Duman: conceptualization (equal); formal analysis (equal); methodology (equal); Project administration (equal); writing review and editing (equal).

\section{References}

1. Farias JA, Frutos F, Esteban A, Casado Flores J, Retta A, Baltodano A, Alía I, Hatzis T, Olazarri F, Petros A, et al. What is the daily practice of mechanical ventilation in pediatric intensive care units? A multicenter study. Intensive Care Med 2004;30(5):918-925.

2. Tekgüç H, Can FK, Şik G, Demirkol D, Özel D, Yaman A, Işgüder R, Yildiztaş D, Yener NA, Öztürk NY, et al. Daily Practice of Mechanical Ventilation and Weaning in Turkish PICUs: A Multicenter Prospective Survey. Pediatr Crit Care Med 2020:E253-E258.

3. Newth CJL, Venkataraman S, Willson DF, Meert KL, Harrison R, Dean JM, Pollack M, Zimmerman J, Anand KJS, Carcillo JA, et al. Weaning and extubation readiness in pediatric patients. Pediatr Crit Care Med 2009;10(1):1-11.

4. Andrew Shanely R, Zergeroglu MA, Lennon SL, Sugiura T, Yimlamai T, Enns D, Belcastro A, Powers SK. Mechanical ventilation-induced diaphragmatic atrophy is associated with oxidative injury and increased proteolytic activity. Am J Respir Crit Care Med 2002;166(10):1369-1374.

5. Kurachek SC, Newth CJ, Quasney MW, Rice T, Sachdeva RC, Patel NR, Takano J, Easterling L, Scanlon M, Musa N, et al. Extubation failure in pediatric intensive care: A multiple-center study of risk factors and outcomes. Crit Care Med 2003;31(11):2657-2664.

6. Kapnadak SG, Herndon SE, Burns SM, Shim YM, Enfield K, Brown C, Truwit JD, Vinayak AG. Clinical outcomes associated with high, intermediate, and low rates of failed extubation in an intensive care unit. J Crit Care 2015;30(3):449-454. 
7. Faustino EVS, Gedeit R, Schwarz AJ, Asaro LA, Wypij D, Curley MAQ, Study R. Accuracy of an extubation readiness test in predicting successful extubation in children with acute respiratory failure from lower respiratory tract disease. Crit Care Med 2017;45(1):94-102.

8. Karl L Yang MJT. A Prospective Study of Indexes Predicting The Outcome of Trials of Weaning From Mechanical Ventilation. N Engl J Med 1992;326.

9. Thiagarajan RR, Bratton SL, Martin LD, Brogan T V., Taylor D. Predictors of successful extubation in children. Am J Respir Crit Care Med 1999;160(5 I):1562-1566.

10. Hubble CL, Gentile MA, Tripp DS, Craig DM, Meliones JN, Cheifetz IM. Deadspace to tidal volume ratio predicts successful extubation in infants and children. Crit Care Med 2000;28(6):2034-2040.

11. Goligher EC, Fan E, Herridge MS, Murray A, Vorona S, Brace D, Rittayamai N, Lanys A, Tomlinson G, Singh JM, et al. Evolution of diaphragm thickness during mechanical ventilation: Impact of inspiratory effort. Am J Respir Crit Care Med 2015;192(9):1080-1088.

12. Jung B, Constantin JM, Rossel N, Le Goff C, Sebbane M, Coisel Y, Chanques G, Futier E, Hugon G, Capdevila $\mathrm{X}$, et al. Adaptive support ventilation prevents ventilator-induced diaphragmatic dysfunction in piglet: An in vivo and in vitro study. Anesthesiology 2010;112(6):1435-1443.

13. Vassilakopoulos T, Petrof BJ. Ventilator-induced Diaphragmatic Dysfunction. Am J Respir Crit Care Med 2004;169(3):336-341.

14. Turton P, ALAidarous S, Welters I. A narrative review of diaphragm ultrasound to predict weaning from mechanical ventilation: where are we and where are we heading? Crit Ultrasound J 2019;11(1):1-7.

15. Li C, Li X, Han H, Cui H, Wang G, Wang Z. Diaphragmatic ultrasonography for predicting ventilator weaning. Med (United States) 2018;97(22).

16. Valverde Montoro D, García Soler P, Hernández Yuste A, Camacho Alonso JM. Ultrasound assessment of ventilator-induced diaphragmatic dysfunction in mechanically ventilated pediatric patients. Paediatr Respir Rev 2021;40:58-64.

17. Lee EP, Hsia SH, Hsiao HF, Chen MC, Lin JJ, Chan OW, Lin CY, Yang MC, Liao SL, Lai SH. Evaluation of diaphragmatic function in mechanically ventilated children: An ultrasound study. PLoS One 2017;12(8):111.

18. Xue Y, Zhang Z, Sheng C-Q, Li Y-M, Jia F-Y. The predictive value of diaphragm ultrasound for weaning outcomes in critically ill children. 2019:1-8.

19. Goligher EC, Brochard LJ, Sebastien-bolz S, Rubenfeld GD, Kavanagh BP, Ferguson ND. Measuring diaphragm thickness with ultrasound in mechanically ventilated patients : feasibility, reproducibility and validity. Intensive Care Med 2015;41(4):642-649.

20. Massimo Zambon, Paolo Beccaria, Jun Matsuno, Marco Gemma, Elena Frati, Sergio Colombo, Luca Cabrini, Giovanni Landoni AZ. Mechanical Ventilation and Diaphragmatic Atrophy in Critically Ill Patients: An Ultrasound Study. Pediatr Crit Care Med Crit Care 2016;44(7):1347-1352.

21. Dinino E, Gartman EJ, Sethi JM, McCool FD. Diaphragm ultrasound as a predictor of successful extubation from mechanical ventilation. Thorax 2014;69(5):423-427.

22. Zhou P, Zhang Z, Hong Y, Cai H, Zhao H, Xu P, Zhao Y, Lin S, Qin X, Guo J, et al. The predictive value of serial changes in diaphragm function during the spontaneous breathing trial for weaning outcome: A study protocol. BMJ Open 2017;7(6):1-6.

23. Şık N, Çitlenbik H, Öztürk A, Yılmaz D, Duman M. Point of care diaphragm ultrasound in acute bronchiolitis: A measurable tool to predict the clinical, sonographic severity of the disease, and outcomes. Pediatr Pulmonol 2021;56(5):1053-1059. 
24. Karthika M, Al Enezi FA, Pillai L V., Arabi YM. Rapid shallow breathing index. Ann Thorac Med 2016;11(3):167-176.

25. Manczur TI, Greenough A PD. Comparison of predictors of extubation from mechanical ventilation in children. Pediatr Crit Care Med 2000;1:28-32.

26. Yoo JW, Lee SJ, Lee JD, Kim HC. Comparison of clinical utility between diaphragm excursion and thickening change using ultrasonography to predict extubation success. Korean J Intern Med 2018;33(2):331339 .

27. IJland MM, Lemson J, van der Hoeven JG, Heunks LMA. The impact of critical illness on the expiratory muscles and the diaphragm assessed by ultrasound in mechanical ventilated children. Ann Intensive Care 2020;10(1).

28. Yaman A, Kendirli T, Ödek Ç, Ateş C, Taşyapar N, Güneş M, Ince E. Efficacy of noninvasive mechanical ventilation in prevention of intubation and reintubation in the pediatric intensive care unit.J Crit Care $2016 ; 32: 175-181$.

\section{Hosted file}

Tables.docx available at https://authorea.com/users/460170/articles/556216-point-of-carediaphragm-ultrasound-in-mechanically-ventilated-children-a-predictive-tool-to-detectextubation-failure
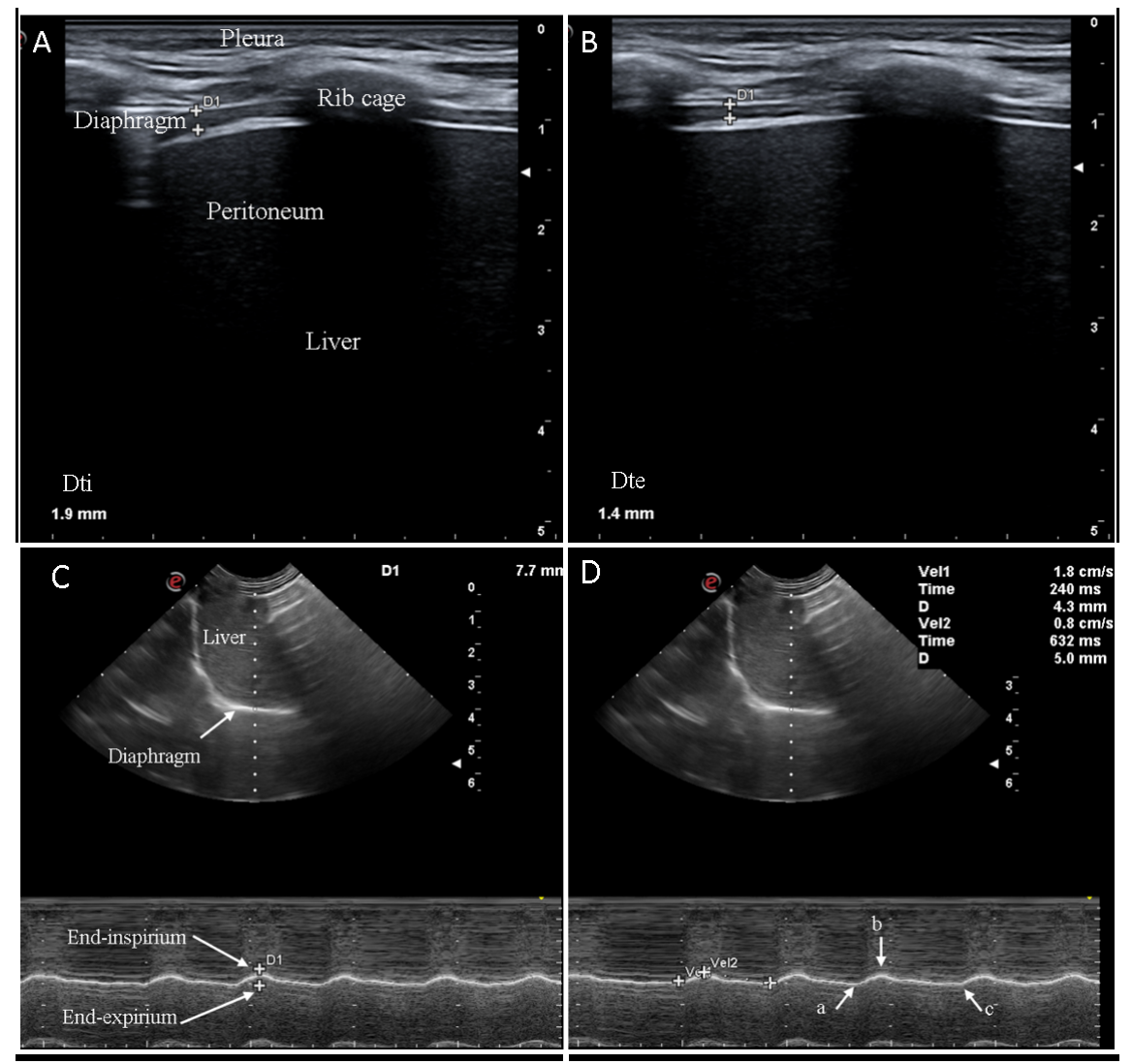


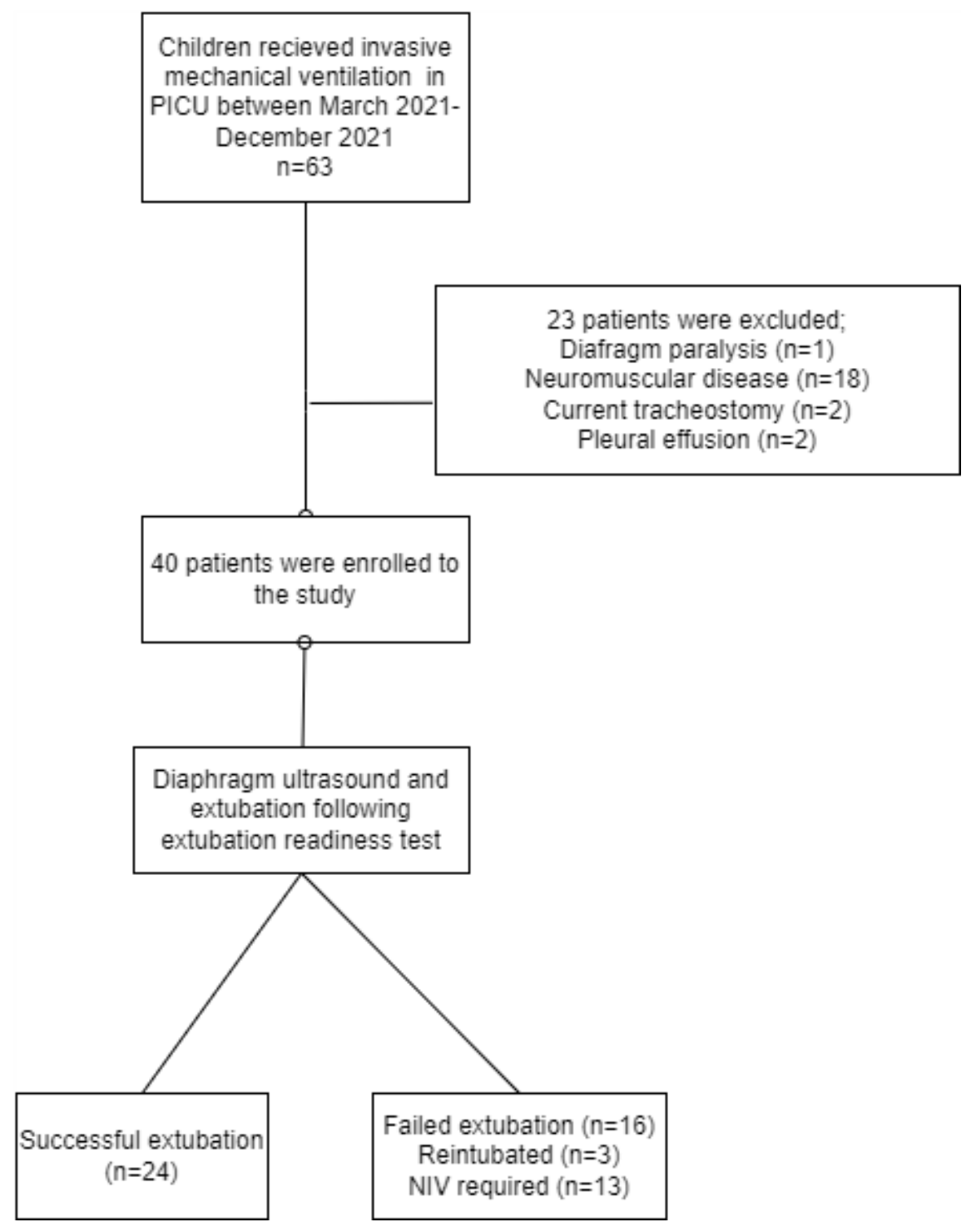




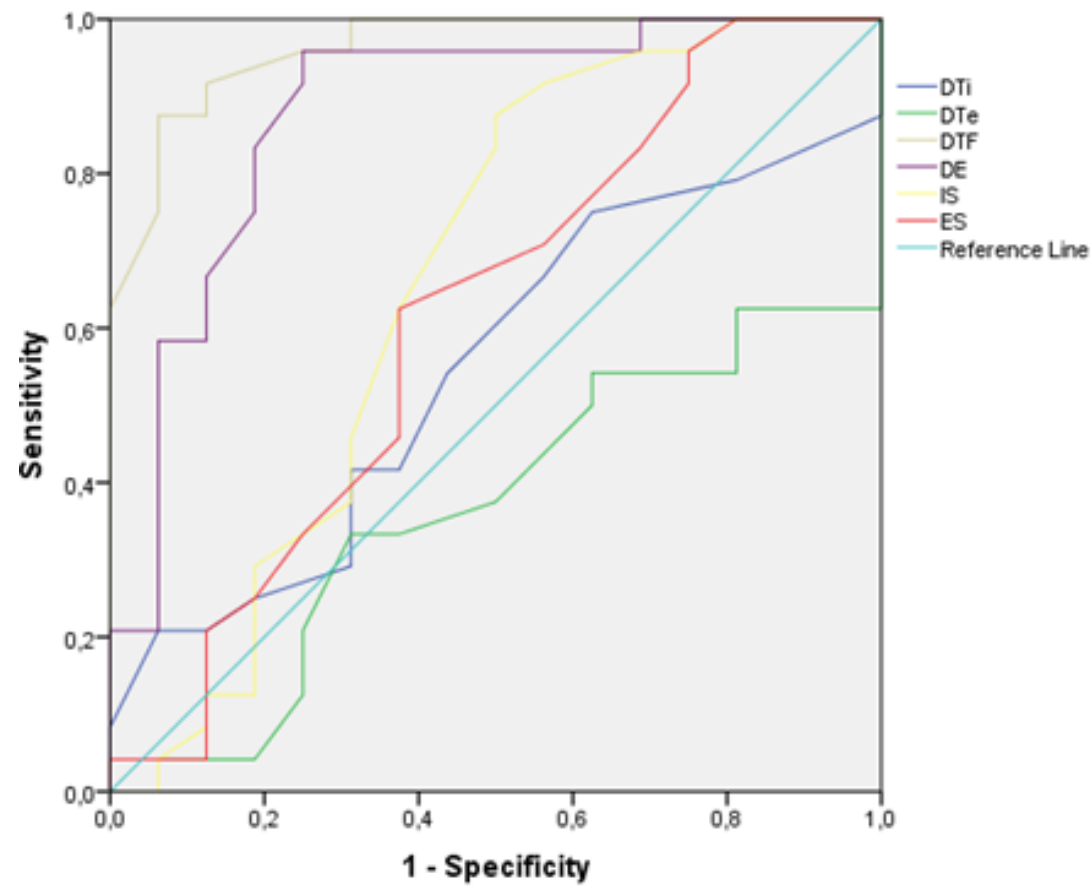

